\title{
A FACTORIZATION THEORY FOR POLYNOMIALS IN $x$ AND IN FUNCTIONS $e^{\alpha x}$
}

BY L. A. MACCOLL

1. Introduction. In this note we consider the problem of determining all representations of a function of the form

$$
f(x)=\sum_{n=0}^{N} \Phi_{n}(x) e^{\alpha_{n} x},
$$

where the $\Phi$ 's are polynomials and the $\alpha$ 's are constants, as a product of functions of the same form. The case in which the $\Phi$ 's are constants has been discussed by J. F. Ritt.* As would be expected, the solution of the general problem possesses some features that are rather different from those appearing in the special case.

It is assumed, of course, that no one of the $\Phi$ 's is identically zero, and that if $N>0$, no two of the $\alpha$ 's are equal. The case of chief interest is that in which $N>0$ and in which the $\Phi$ 's have no common zero. The discussion will be confined to this case. We select those of the $\alpha$ 's for which the real parts are least, and of the constants so selected (if there be more than one) we select the one for which the coefficient of $(-1)^{1 / 2}$ is least. Let the constant so selected be denoted by $\alpha_{0}$. We assume that $\alpha_{0}=0$. The class of functions of the form (1) satisfying the conditions stated in this paragraph will be called $C$.

If $f(x), f_{1}(x), \cdots, f_{s}(x)$ are all of the form (1), and if $f(x)=f_{1}(x) \cdots f_{s}(x)$, we shall say that $f(x)$ is divisible by each of the functions $f_{i}(x)$, and each of the latter functions will be called a factor of $f(x)$. A function which is divisible only by itself and by functions of the form $A e^{\alpha x}$, where $A$ and $\alpha$ are constants, will be called irreducible.

2. Reduction to a Problem Concerning Polynomials. Monomial factors of $f(x)$ are, in a certain sense, trivial. Henceforth we consider only factors having at least two terms. These factors may be taken as belonging to the class $C$.

Suppose that the function

* J. F. Ritt, $A$ factorization theory for functions $\sum_{i=1}^{n} a_{i} e^{\alpha_{i x}}$, Transactions of this Society, vol. 29 (1927), pp. 584-596. 


$$
g(x)=\sum_{p=0}^{P} \Psi_{p}(x) \epsilon^{\beta_{p} x}
$$

is a factor of $f(x)$. Ritt has shown in effect that each $\beta$ is a linear combination of the $\alpha$ 's with rational coefficients. He has also shown the existence of a set of numbers $\rho_{1}, \cdots, \rho_{q}$, which are linearly independent with respect to rational coefficients, and which are such that each $\alpha$ is a linear combination of the $\rho$ 's with non-negative integral coefficients. Each $\beta$ is a linear combination of the $\rho$ 's with non-negative rational coefficients.

Consider a representation of $f(x)$ as a product of factors,

$$
f(x)=\sum_{n=0}^{N} \Phi_{n}(x) e^{\alpha_{n} x}=\prod_{s=1}^{S} \sum_{p=0}^{P_{s}} \Psi_{s p}(x) e^{\beta_{s p} x} .
$$

The $\alpha$ 's and $\beta$ 's in this equation are understood to be expressed in terms of the $\rho$ 's as explained above. In each $\Phi$ and $\Psi$ in (2) we replace $x$ by the indeterminate $y_{0}$, and we replace each function $\exp \left(k \rho_{i} x\right)$, where $k$ is a non-negative rational number, by $y_{i}{ }^{k}$. Equation (2) is thus replaced by a relation in the $y$ 's which is easily seen to be an identity. Each of the indeterminates $y_{1}, \cdots, y_{q}$ can be replaced by a positive integral power of itself in such a way that the right-hand member of the relation in the $y$ 's obtained from (2) becomes a polynomial in the $y$ 's. The relation thus obtained is an identity.

We now have a method for factoring $f(x)$. First we express the $\alpha$ 's as linear combinations, with non-negative integral coefficients, of numbers $\rho_{1}, \cdots, \rho_{q}$ which are linearly independent with respect to rational coefficients. In each of the polynomials $\Phi_{n}$ we replace $x$ by $y_{0}$, and we replace $\exp \left(\rho_{i} x\right)$ by $y_{i}$, thus obtaining a polynomial $Q\left(y_{0}, y_{1}, \cdots, y_{q}\right)$. In this polynomial we replace the indeterminates $y_{i},(i>0)$, in all possible ways by positive integral powers of themselves, thus obtaining a family of polynomials $Q\left(y_{0}, y_{1} t_{1}, \cdots, y_{q} t_{q}\right)$. To each resolution of each of these polynomials into factors there corresponds a factorization of $f(x)$. All factorizations of $f(x)$ are obtained in this way.

3. The Problem Concerning Polynomials. Because of the conditions that we have imposed on $f(x)$, the polynomial $Q$ has no non-constant monomial factor. Let $Q_{1}\left(y_{0}, y_{1}, \cdots, y_{v}\right)$ be an irreducible factor of $Q$. We shall consider the question: For which 
positive integers $t_{i}$ is the polynomial $Q_{1}\left(y_{0}, y_{1} t_{1}, \cdots, y_{\nu} t_{\nu}\right)$ reducible? This is a modification of a problem studied by Ritt in his paper, and later by E. Gourin, who simplified the proofs and obtained stronger results. ${ }^{*}$ The modification lies in the fact that, whereas in the problem of Ritt and Gourin all of the variables enjoy the same status, in our problem we may have one variable, $y_{0}$, that is exceptional in that it is not replaceable by a power of itself.

If $Q_{1}$ is independent of $y_{0}$, we have the case considered by Gourin. Henceforth we assume that $Q_{1}$ depends on $y_{0}$.

Suppose that $Q_{1}$ has at least three terms. By Gourin's theory, if there exists a set of positive integral $t$ 's such that the polynomial $Q_{1}\left(y_{0} t_{0}, y_{1} t_{1}, \cdots, y_{\nu} t_{\nu}\right)$ is reducible, there exists one and only one finite aggregate of sets of positive integers

$$
t_{10}, t_{11}, \cdots, t_{1 v} ; \cdots ; t_{M 0}, t_{M 1}, \cdots, t_{M v}
$$

having the following properties. (1) For each $m$, the polynomial $Q_{1}\left(y_{0}{ }^{t_{m 0}}, \cdots, y_{v}{ }^{t_{m \nu}}\right)$ is reducible. (2) If $Q_{1}\left(y_{0}{ }^{t_{0}}, \cdots, y_{\nu}{ }^{t_{\nu}}\right)$ is reducible, there exists in the aggregate (3) one and only one set, say $t_{j 0}, \cdots, t_{j \nu}$, such that each $t_{i}$ is an integral multiple of $t_{j i}$, say $t_{i}=\delta_{i} t_{j i}$, and the irreducible factors of $Q_{1}\left(y_{0}{ }^{t_{0}}, \cdots, y_{\nu}{ }^{t_{\nu}}\right)$ are obtained by replacing each $y_{i}$ by $y_{i}{ }_{i}^{\delta_{i}}$ in the irreducible factors of $Q_{1}\left(y_{0}{ }^{t_{j 0}}, \cdots, y_{v}{ }^{t}{ }_{j v}\right)$.

If no one of the numbers $t_{m 0}$ is unity, there exists no set of $t$ 's such that $Q_{1}\left(y_{0}, y_{1}{ }^{t_{1}}, \cdots, y_{v} t_{\nu}\right)$ is reducible. Now suppose that $t_{10}=t_{20}=\cdots=t_{\mu 0}=1$, and that $t_{m 0} \neq 1$ for $m>\mu$. Then the sets

$$
t_{11}, \cdots, t_{1 v} ; \cdots ; t_{\mu 1}, \cdots, t_{\mu \nu}
$$

have the following properties. (1) For each $m, 1 \leqq m \leqq \mu$, $Q_{1}\left(y_{0}, y_{1} t_{m 1}, \cdots, y_{v} t_{m v}\right)$ is reducible. (2) If $Q_{1}\left(y_{0}, y_{1} t_{1}, \cdots, y_{\nu} t_{\nu}\right)$ is reducible, there exists in (4) one and only one set, say $t_{j 1}, \cdots, t_{j \nu}$, such that each $t_{i}$ is an integral multiple of $t_{j i}$, say $t_{i}=\delta_{i} t_{j i}$, and the irreducible factors of $Q_{1}\left(y_{0}, y_{1} t_{1}, \cdots, y_{v} t_{v}\right)$ are obtained by replacing $y_{1}, \cdots, y_{\nu}$ by $y_{1} \delta_{1}, \cdots, y_{\nu} \delta_{\nu}$, respectively, in the irreducible factors of $Q_{1}\left(y_{0}, y_{1} t_{j 1}, \cdots, y_{\nu}{ }^{t_{j \nu}}\right)$. The

* E. Gourin, On irreducible polynomials in several variables which become reducible when the variables are replaced by powers of themselves, Transactions of this Society, vol. 32 (1930), pp. 485-501. 
aggregate (4) is the only aggregate of sets having these two properties.

Now suppose that $Q_{1}$ has only two terms, so that, with a proper assignment of the subscripts, it is of the form

$$
Q_{1}=a y_{0}^{\lambda_{0}} y_{1}^{\lambda_{1}} \cdots y_{r}^{\lambda_{r}}+b y_{r+1}^{\lambda_{r+1}} \cdots y_{s}^{\lambda_{s}},
$$

where $a$ and $b$ are constants, and the $\lambda$ 's are positive integers. By Gourin's theory, the infinite system of sets of positive integers

$$
t_{20}, t_{21}, \cdots, t_{2 v} ; t_{30}, t_{31}, \cdots, t_{3 v} ; \cdots,
$$

where $t_{k i}=k / d_{k i}, d_{k i}$ being the greatest common divisor of $\lambda_{i}$ and $k$, has the following properties. (1) For each $k>1$, the polynomial $Q_{1}\left(y_{0} t_{k 0}, \cdots, y_{\nu}{ }^{t_{k \nu}}\right)$ is reducible. (2) If $Q_{1}{ }^{(t)}=$ $Q_{1}\left(y_{0} t_{0}, \cdots, y_{\nu} t_{\nu}\right)$ is reducible, there exists in (5) one and only one set, say $t_{j 0}, \cdots, t_{j \nu}$, such that each $t_{i}$ is an integral multiple of $t_{j i}$, say $t_{i}=\delta_{i} t_{j i}$, and the irreducible factors of $Q_{1}{ }^{(t)}$ are obtained by replacing each $y_{i}$ by $y_{i}{ }_{i} i$ in the irreducible factors of $Q_{1}\left(y_{0} t_{j_{0}}, \cdots, y_{v}{ }^{t_{i v}}\right)$. The system (5) is the only system of sets of integers having these two properties.

If no one of the numbers $t_{20}, t_{30}, \cdots$ is unity, there is no set of positive integral $t$ 's for which $Q_{1}\left(y_{0}, y_{1}{ }^{t_{1}}, \cdots, y_{v} t_{\nu}\right)$ is reducible. Now suppose that $t_{m_{1} 0}=t_{m_{2} 0}=\cdots=1$, and that the remaining numbers of the set $t_{20}, t_{30}, \cdots$ are all different from unity. Then the system of sets

$$
t_{m_{1} 1}, \cdots, t_{m_{1} \nu} ; t_{m_{2} 1}, \cdots, t_{m_{2} \nu} ; \cdots
$$

has the following properties. (1) For any set of (6) the polynomial $Q_{1}\left(y_{0}, y_{1}{ }^{t_{m_{h} 1}}, \cdots, y_{\nu}{ }^{t_{m_{h}}}\right)$ is reducible. (2) If for any positive integral $t$ 's the polynomial $Q_{1}\left(y_{0}, y_{1}{ }^{t_{1}}, \cdots, y_{\nu} t_{v}\right)$ is reducible, there exists in (6) one and only one set, say $t_{m_{j}}, \cdots$, $t_{m_{j} \nu}$, such that each $t_{i}$ is an integral multiple of $t_{m_{j} i}$, say $t_{i}=\delta_{i} t_{m_{j} i}$, and the irreducible factors of $Q_{1}\left(y_{0}, y_{1}^{t_{1}}, \cdots, y_{\nu} t_{\nu}\right)$ are obtained by replacing $y_{1}, \cdots, y_{v}$ by $y_{1}^{\delta_{1}}, \cdots, y_{\nu} \delta_{\nu}$, respectively, in the irreducible factors of $Q_{1}\left(y_{0}, y_{1} t_{m_{j}}, \cdots, y_{v}{ }^{\left.t_{m_{j}} \nu\right)}\right.$. The system (6) is the only system of sets of positive integers having these two properties.

The system (6) does not exist if $\lambda_{0}=1$; if $\lambda_{0}>1$, the system exists, the integers $m_{1}, m_{2}, \cdots$ being precisely the divisors of $\lambda_{0}$ that are greater than 1 . If the system (6) exists, the number of 
sets in it is finite. This is the outstanding novelty introduced by the presence of the exceptional variable $y_{0}$.

4. The Factorization Theorem. We are now ready to complete the solution of our problem. We begin by resolving $Q$ into its irreducible factors. The irreducible factors which do not contain $y_{0}$ are treated as in Ritt's theory. Those which contain at least three terms give rise to irreducible factors of $f(x)$. Those which have only two terms give rise to a definite set of factors of the form $b_{0}+\sum_{i=1}^{m} b_{i} \exp \left(\beta_{i} x\right)$, where the $b^{\prime}$ 's are constants, the $\beta$ 's in each function have rational ratios to one another, and any two $\beta$ 's in different functions have an irrational ratio. Ritt calls these factors simple functions. A simple function has an infinite set of factors.

Consider an irreducible factor of $Q$, say $Q_{1}$, which contains $y_{0}$. We have seen that either there are no positive integral $t$ 's for which $Q_{1}\left(y_{0}, y_{1}{ }^{t_{1}}, \cdots, y_{v}{ }^{t v}\right)$ is reducible, or there exists one and only one finite aggregate of sets of positive integers

$$
t_{m 1}, \cdots, t_{m \nu}, \quad(m=1, \cdots, M),
$$

which have the following properties. (1) For each value of $m$, $Q_{1}\left(y_{0}, y_{1}{ }^{t_{m 1}}, \cdots, y_{v}{ }^{t_{m \nu}}\right)$ is reducible. (2) If $Q_{1}\left(y_{0}, y_{1}{ }^{t_{1}}, \cdots, y_{v}{ }^{t_{\nu}}\right)$ is reducible, there is one and only one value of $m$, say $\mu$, such that each $t_{i}$ is an integral multiple of $t_{\mu i}$, say $t_{i}=\delta_{i} t_{\mu i}$, and the irreducible factors of $Q_{1}\left(y_{0}, y_{1}{ }^{t_{1}}, \cdots, y_{\nu} t_{\nu}\right)$ are obtained by replacing $y_{1}, \cdots, y_{\nu}$ by $y_{1}^{\delta_{1}}, \cdots, y_{\nu}^{\delta_{\nu}}$, respectively, in the irreducible factors of $Q_{1}\left(y_{0}, y_{1}{ }^{t_{\mu 1}}, \cdots, y_{\nu}{ }^{t_{\mu \nu}}\right)$.

If no set of $t^{\prime}$ s exists such that $Q_{1}\left(y_{0}, y_{1}{ }^{t_{1}}, \cdots, y_{v}{ }^{t_{v}}\right)$ is reducible, the function $Q_{1}\left(x, e^{\rho_{1} x}, \cdots, e^{\rho_{\nu} x}\right)$ is an irreducible factor of $f(x)$.

If a set of $t^{\prime}$ s exists such that $Q_{1}\left(y_{0}, y_{1}{ }_{1}, \cdots, y_{v}{ }_{\nu}\right)$ is reducible, we have a relation of the form (in the notation used above)

$$
Q_{1}\left(y_{0}, y_{1}^{t_{m 1}}, \cdots, y_{\nu} t_{m \nu}\right)=\prod_{k=1}^{K_{m}} Q_{1 k}\left(y_{0}, y_{1}, \cdots, y_{\nu}\right),
$$

where the $Q$ 's in the second member are irreducible. Let the value of $m$ be selected so that $K_{m}$ has its maximum value. Then each of the functions

$$
Q_{1 k}\left(x, e^{\rho_{1} x / t_{m 1}}, \cdots, e^{\rho_{\nu} x / t_{m \nu}}\right), \quad\left(k=1, \cdots, K_{m}\right),
$$

is irreducible. In fact if this were not so, there would exist a 
set of positive integers $\delta_{i}$ such that $Q_{1}\left(y_{0}, y_{1} \delta_{1}, \cdots, y_{v} \delta_{\nu}\right)$ would be resolvable into more than $K_{m}$ factors, which is not the case. Each of the functions (7) is a factor of $f(x)$.

When we multiply together the simple functions coming from the irreducible binomial factors of $Q$ which do not involve $y_{0}$ and the irreducible functions coming from the remaining irreducible factors of $Q$, we have a resolution of $f(x)$ into factors belonging to the class $C$. It is easily seen that this factorization is unique. Thus we have the following theorem.

Theorem. A function $f(x)$ belonging to the class $C$ can be expressed in one and only one way as a product

$$
f(x)=I_{1}(x) \cdots I_{m}(x) S_{1}(x) \cdots S_{n}(x),
$$

where each factor belongs to $C$, the I's are irreducible functions, and the $S$ 's are simple functions, $b_{0}+\sum b_{i} \exp \left(\beta_{i} x\right)$, such that the ratio of any two $\beta$ 's in different functions is irrational.

Bell Telephone Laboratories

\section{THE NUMBER OF TRISECANTS OF A SPACE CURVE OF ORDER $m$ WHICH MEET AN $i$-FOLD SECANT*}

BY L. A. DYE

The number of trisecants of a space curve $C_{m}$, of order $m$, which meet a general line was determined by Zeuthen, $\dagger$ but if the line happens to be an $i$-fold secant, $i>2$, it lies on the ruled surface of trisecants and the formula fails. In algebraic geometry some extension of Zeuthen's work to cover this neglected case is often necessary, so by means of a correspondence we show that the number of trisecants of a $C_{m}$ which meet an $i$-fold secant $l$ is

$(m-2)[h-m(m-1) / 6]-i(h-m+2)+i(i-1)(i-2) / 6$, where $h$ is the number of apparent double points of $C_{m}$.

In the plane determined by $l$ and one of the $h^{\prime}=h-i(i-1) / 2$

* Presented to the Society, October 27, 1934.

$\dagger$ H. G. Zeuthen, Sur les singularités des courbes gauches, Annali di Matematica, (2), vol. 3 (1869), pp. 175-217. 